\title{
DIE IM NACHFELD STEHENDEN SÄTZE MIT SPITZENSTELLUNG DES FINITEN VERBS
}

ABSTRACT: The causal clauses with the initial position of the predicate

The paper discusses causal clauses in contemporary German, positioned after the main clause, which lack a conjunction combining it with that clause. Their characteristic feature is the initial position of the predicate and the obligatory use of the particle doch. Numerous grammars either ignore such causal clauses or consider them obsolete, although they are found not only in literary but also in colloquial speech texts.

KEYwORDS: causal clauses; initial position of the predicate; German grammars

Gegenstand meiner Analyse sind die im Nachfeld stehenden Sätze mit Spitzenstellung des finiten Verbs, in denen zusätzlich obligatorisch die Partikel doch stehen muss. Solche Sätze stehen nicht im Zentrum des Interesses der deutschen Grammatiker. In vielen Grammatiken werden sie einfach außer Acht gelassen. Sie unterscheiden sich von den Satzverbindungen, in denen zwei selbständige Hauptsätze asyndetisch miteinander verknüpft werden:

Die Kinder spielen im Garten, die Eltern sitzen vor dem Fernseher.

Bei den Satzverknüpfungen, in denen das finite Verb im zweiten Satz in der Spitzenposition steht, handelt es sich um einen abhängigen Satz, der auf den Sachverhalt des ersten Satzes Bezug nimmt und ihn auf eine bestimmte Weise begründet:

Sie (eine Neuentwicklung) beendet das Monopol der Boeing 747 bei zivilen Großflugzeugen, bietet sie doch 555 Personen auf zwei „Stockwerken“ Platz. (Der Standard, 28.04.2005, S. 5)

Das will der engagierte Fernsehreporter mittels einer brisanten Enthüllungsstory verhindern, ist er doch davon überzeugt, dass Hunters makellose Verurteilungsrate darauf beruht, dass dieser Beweise manipuliert. (Treffpunkt Kino Cinemaxx, 2010, Februar, S. 19)

Józef Wiktorowicz - Uniwersytet Warszawski, Warszawa, j.wiktorowicz@uw.edu.pl 
Aus diesem Grund werden solche uneingeleiteten, im Nachfeld stehenden Sätze zusammen mit den eingeleiteten Kausalsätzen behandelt. In den meisten Grammatiken der deutschen Gegenwartssprache werden jedoch solche uneingeleiteten Kausalsätze mit der Spitzenstellung des finiten Verbs und der obligatorischen Partikel doch gar nicht erwähnt, als ob sie in der deutschen Gegenwartssprache gar nicht existierten. In der Duden-Grammatik findet man keine Angaben zu den uneingeleiteten Kausalsätzen; auch in der Grammatik von Helbig/Buscha findet man keine Informationen über die im Nachfeld stehenden Sätze mit der Spitzenstellung des finiten Verbs. Erst in den Grundzügen einer deutschen Grammatik von K. E. Heidolph, W. Flämig u. a. findet man im Kapitel „Kausalverhältnisse“ (Heidolph u. a. 1984: §54-56) Informationen über verschiedene subordinierende Konjunktionen, die einen begründenden Satz mit Endstellung der finiten Verbform einleiten. Und im Zusammenhang mit den Kausalsätzen, die durch die Konjunktion zumal eingeleitet werden, wird erwähnt, dass diese Konjunktion durch die Elemente wo und die Partikel doch ersetzt werden kann. Weiter wird ausgeführt: „Unter Eliminierung des einleitenden wo bzw. $d a$ tritt im begründenden Nachsatz die finite Verbform aus der End- in die Spitzenposition, und der so formierte Spitzenstellungssatz mit obligatorischem doch im Satzinnern erfüllt die gleiche Funktion wie etwa ein Endstellungssatz mit einleitendem zumal" (Heidolph u. a. 1984: 801, §56). Diese Ausführungen wurden durch die folgenden Beispielsätze erläutert:

Er erhielt die Stellung, zumal er der einzige Spezialist unter den Bewerbern war.

Er erhielt die Stellung, war er doch der einzige Spezialist unter den Bewerbern.

(Heidolph u. a. 1984: 801)

Auch in der Deutschen Grammatik von Ulrich Engel (1988: 269) wird auf die abhängigen Sätze mit Spitzenstellung des finiten Verbs und der Partikel doch eingegangen. Die von Engel als ,uneingeleitete Kausalsätze“ bezeichneten Sätze werden wie folgt erläutert:

Sie sind in die Nähe der wo-Sätze zu stellen; wie diese verlangen sie meist die Abtönungspartikel doch. Uneingeleitete Kausalsätze nennen einen Grund, der entweder allgemein akzeptiert ist oder den jeder akzeptieren sollte:

Er kam gerne in die Stadt zurück, war ihm doch aus seiner Studienzeit jede Straße um den Alten Markt vertraut.

Im Gegensatz zu den kausalen wo-Sätzen sind die nicht eingeleiteten Kausalsätze ausschließlich der Schriftsprache vorbehalten; sie gelten allerdings auch hier als zunehmend veraltet.“(Engel 1988: 269)

Ulrich Engel betont in seiner Grammatik, dass solche abhängigen Sätze ohne Konjunktion und mit der Spitzenstellung des finiten Verbs eine Besonderheit der Schriftsprache wären und dass sie als „zunehmend veraltet“ gelten. Solche Behauptungen 
entsprechen aber nicht der sprachlichen Wirklichkeit, was im weiteren Teil meines Beitrags gezeigt wird.

Auch in der dreibändigen Grammatik der deutschen Sprache von G. Zifonun u. a. werden die uneingeleiteten Kausalsätze kurz behandelt. Im Kap. 7.3.4.2 „Die Kausalen Subjunktoren und die peripheren Kausalsätze“ (Zifonun u. a. 1997: 2298f.) werden zunächst die kausalen Subjunktoren $d a$ und weil behandelt, dann wird auf die zumal-Sätze eingegangen. Zur Peripherie der kausalen Nebensätze werden in der IdS-Grammatik wo-Sätze mit der obligatorischen Partikel doch gerechnet sowie ,im Nachfeld befindliche abhängige Verberstsätze ebenfalls mit der Partikel doch.“ (Zifonun u. a. 1997: 2299). Der Gebrauch der peripheren Kausalsätze wird mit folgenden Beispielen erläutert:

Lohnt sich denn die neue Vorschrift, wo doch nur ein Viertel des Angebotes im Handel Ex-und-hoppWare ist. (Zeit, 1.3. 1985, 32)

Viel ist es nicht, was der Außenminister mitbringen kann, ist doch der Handlungsspielraum der deutschen Politik äußerst eingeschränkt. (Heute Journal, 12.2. 1991) (Zifonun u. a. 1997: 2299)

Die Funktion der wo-Sätze und der uneingeleiteten Kausalsätze mit der Spitzenstellung des finiten Verbs wird in der IdS-Grammatik wie folgt erläutert: „Die beiden peripheren Formen werden verwendet, um stützende Argumente und Plausibilisierungen für das Gesagte oder die vollzogene Sprechhandlung nachzuschieben. In der Regel wird man sie als Kommentierungen auf der Ebene des Modus dicendi einordnen.“ (Zifonun u. a. 1997: 2299). In der Ids-Grammatik wird nicht mehr behauptet, dass die uneingeleiteten Kausalsätze veraltet seien. Auf Grund ihres seltenen Auftretens werden sie zur Peripherie gerechnet, sie werden aber nicht als veraltet betrachtet, weil solche Sätze nicht nur im 20. Jahrhundert, sondern auch im 21. Jahrhundert ein fester Bestandteil des deutschen Sprachsystems sind.

Die abhängigen Sätze mit der Spitzenstellung des finiten Verbs und der Partikel doch treten in den deutschen Texten - entgegen den Behauptungen von Ulrich Engel zu Beginn des 21. Jahrhunderts relativ oft auf. Die Suche nach entsprechenden Belegen ist schwierig, dennoch lassen sich solche Sätze in der deutschen Literatur und auch in der Presse finden. Im Roman von Günter Grass Grimms Wörter findet man vier Sätze mit der Spitzenstellung des finiten Verbs und der Partikel doch:

Fallersleben war mit den Grimmbrüdern seit Jahren bekannt, teilte er doch deren Neigung zur Erforschung frühdeutscher Dichtung. (Grimms Wörter 111)

Der Autor begründet die Tatsache, dass Grimm-Brüder Fallersleben kannten, mit der Tatsache, dass alle drei Männer die gleichen Interessen hatten, und zwar die Erforschung der frühdeutschen Dichtung. Ähnlich wird in einem anderen Satz begründet, warum Jacob Grimm der Erbadel suspekt war: 
Ihm (Jacob Grimm) war der Erbadel schon seit Kasseler Schuljahren suspekt, wurden doch blaublütige Söhnchen von den Lehrern mit Sie angesprochen, den Brüdern kam jedoch nur demütigend ein Er zu. (Grimms Wörter 159)

In den Grundzügen einer deutschen Grammatik werden die uneingeleiteten Kausalsätze in die Nähe der zumal-Sätze gestellt, was meines Erachtens nicht ganz zutreffend ist, weil die zumal-Sätze zum Ausdruck bringen, dass es einige Gründe für den genannten Sachverhalt gibt, der Sprecher aber noch eine zusätzliche wichtige Begründung für den Sachverhalt nennt. Bei den uneingeleiteten Kausalsätzen mit der Spitzenstellung des finiten Verbs und der Partikel doch handelt es sich vielmehr um ein stützendes, offensichtliches Argument für den genannten Sachverhalt:

Und verdunkelt mögen seine Gedanken sein, bedrückt ihn doch seit geraumer Zeit etwas, das die Ärzte Depression nennen. (Grimms Wörter 206,7)

Die trüben Gedanken der Hauptfigur sind das Resultat einer Depression, deshalb versucht der Autor diesen Tatbestand nicht besonders zu begründen.

Die uneingeleiteten Kausalsätze scheinen nicht nur auf die Literatursprache beschränkt zu sein, denn die entsprechenden Belege mit der Spitzenstellung des finiten Verbs und der Partikel doch findet man in den letzten Jahren nicht nur in der schöngeistigen Literatur (z. B. bei Günter Grass), sondern auch in den Kriminalromanen, die sich stärker an die Umgangssprache anlehnen und auch in den Fernsehzeitschriften, in denen bei der kurzen Darstellung des Inhalts der Fernsehfilme der umgangssprachliche Wortschatz bevorzugt wird. Zunächst nenne ich Beispiele aus den Kriminalromanen, die keineswegs als Vertreter der gehobenen Standardsprache angesehen werden können:

Alex war erstaunt, hatte Sergio ihr doch erzählt, sein Bruder sei an den Folgen einer Krankheit gestorben. (Unter Haien 387)

Das Erstaunen der Hauptfigur wird damit begründet, dass sie einen anderen Grund für den Tod des Bruders von Sergio gehört hatte.

Die ganze Angelegenheit entbehrte nicht einer gewissen Ironie, war er doch letztendlich ein betrogener Betrüger. (Wer Wind sät 446)

Auch hier kommentiert die Autorin den genannten Sachverhalt mit der Tatsache, dass eben der Betrüger betrogen wurde.

Auch in den Fernsehzeitschriften, in denen kurze Inhaltsangaben über die Fernsehfilme gebracht werden und der umgangssprachliche Stil und Wortschatz bevorzugt werden, tauchen relativ oft uneingeleitete Kausalsätze mit der Spitzenstellung des finiten Verbs und der Partikel doch:

Nach einem Mord an einem Kneipenbesitzer sieht alles nach einer Beziehungstat aus, hat doch das Opfer seinem Freund Erik einst die Freundin ausgespannt. (TV-Spielfilm 2015, H. 9, S. 42) 
Der Mord als Beziehungstat wird mit der Tatsache begründet, dass das Mordopfer seinem Freund einst die Freundin ausgespannt hat. Ähnlich wird in einem anderen Satz kommentiert, warum die Stadt Bamberg, eine relativ kleine Stadt, kein ruhiges Pflaster für einen Kriminalkommissar ist:

Kommissar Haller (Thomas Schmauser) ist nach Bamberg zurückgekehrt. Kein ruhiges Pflaster, muss er doch mit Kollegin Birgit Sacher den Mord an einer Westernreiterin aufklären. ( $T V$-Spielfilm 2015, H. 20, S. 38)

Man hat den Eindruck, dass gerade in den Fernsehzeitschriften, in denen der knappe Stil bei den Inhaltsangaben der Fernsehfilme bevorzugt wird, die uneingeleiteten Kausalsätze gern verwendet werden.

Solche Sätze mit der Spitzenstellung des finiten Verbs und der obligatorischen Partikel doch, die seit dem Frühneuhochdeutschen bekannt sind, werden in der neuhochdeutschen Periode und auch in der modernen deutschen Standardsprache nach wie vor relativ oft gebraucht. Deshalb sollten sie nicht nur in größeren Grammatiken der deutschen Sprache, sondern auch in den Grammatiken kleineren Umfangs berücksichtigt werden, insbesondere in den DaF-Grammatiken.

\section{Quellennachweise}

Grass, Günter (2012): Grimms Wörter. Eine Liebeserklärung. München: Steidl.

Neuhaus, Nele (2011): Wer Wind sät. Berlin: Ullstein.

Neuhaus, Nele (2013): Unter Haien. Berlin: Ullstein.

Der Standard, 28.04.2005

TV-Spielfilm, 2015, H. 9.

TV-Spielfilm, 2015, H. 20.

Treffpunkt Kino Cinemaxx, 2010, Februar

\section{Literatur}

Dudenredaktion (Hg.) (2009): Duden: Die Grammatik. 8., überarbeitete Aufl. Mannheim/Wien/Zürich: Dudenverlag.

Engel, Ulrich: Deutsche Grammatik. Heidelberg 1988.

Helbig, Gerhard/Buscha, Joachim (1998): Deutsche Grammatik. Ein Handbuch für den Ausländerunterricht. 18. Auflage. Lepizig: Enzyklopädie Verlag.

Heidolph, Karl Erich/Flämig, Walter/Motsch, Wolfgang (1984): Grundzüge einer deutschen Grammatik. Berlin: Akademie Verlag.

Zifonun, Gisela u. a. (1997): Grammatik der deutschen Sprache, Bd. 1-3. Berlin/New York: Walter de Gruyter. 
